\title{
A Fast Adaboosting Based Method for Iris and Pupil Contour Detection
}

\author{
Francisco Silva Mata, Eduardo Garea Llano, Estela María Álvarez Morales, \\ and José Luís Gil Rodríguez
}

Advanced Technology Application Center. 7ma, No. 21812, Siboney, Playa, Cuba 12200

\{fjsilva, egarea, ealvarez, jlgil\}@cenatav.co.cu

\begin{abstract}
The iris localization plays a fundamental role in the recognition process because the speed and performance of the iris recognition system largely depends on the quality of the pupil and iris detection. This process includes the detection of inner (pupil) and outer (iris) boundaries. In this paper we present a new method for iris and pupil boundaries detection based on Adaboosting technique for localization of circular objects and an algorithm based on the elements of analytic geometry, in particular, the determination of the bounded circumference of a tangential square that encloses the pupil and iris. The proposed approach overcomes the limitations that had previous methods regarding the use of images obtained under not controlled conditions like specular light reflected in the pupil or in the iris. We experimented our approach comparing the results in detection with the results obtained by Daugman algorithm using images from two contrasting databases, CASIA and UBIRIS.
\end{abstract}

\section{Introduction}

Recognition using the iris texture has been an active investigation field in last years; it has been considered the better phenotypic feature in human face that determines its identity and offers biometric feature acquisition without invasion. Recognition of a person by iris constitutes one of the main applications of the biometrics at present time. The first step in the recognition process is the automatic iris localization. The human eye iris is characterized by a circular or quasi circular form limited by two borders (iris inner border and outer). The iris inner border coincides with the contour of the eye's pupil and the iris outer border establishes the contact iris-sclera (Fig.1). A number of algorithms have been proposed for automatic iris contour detection to obtain both, the iris inner and outer border parameters in order to isolate the iris texture information. [1-7]. The limitations of these algorithms are the fact that they were thought for working using images taken under well controlled illumination conditions [8] where of the specular light reflected in the pupil or in the iris does not represent a problem. The high computational cost is another of their limitations.

In this paper we present a method based on Viola and Johns algorithms [9] that localized the pupil and iris circles. The results of this process are the localization of 
the iris inner and outer border enclosed in a tangential to border square. The next step is to draw the circle enclosed in the detected square that describes the iris and pupil borders. We experimented our approach using images from UBIRIS database that incorporates images with several noise factors, thus permitting the evaluation of robustness iris recognition methodologies and compared the results with those obtained using images taken in well controlled environment of CASIA database.

\section{Automatic Detection of Tangential Square to Pupil and Iris Borders}

Detection of tangential square to pupil and iris borders is achieved through the algorithm of Viola and Johns [9], implemented in the OpenCV library [10]. The advantages that this algorithm offers are several: The image representation called integral image, which allows a very quickly computation of the features used by the detectors. The learning algorithm is based on the Stump-based $24 \times 24$ Gentle Adaboost, which lets the selection of a small number of features from the initial set and to obtain efficient classifiers. This method combines classifiers increasingly in a cascade of simple classifiers for discriminate particular features [9].

We trained two cascade detectors, the first devoted to detect the pupil circle (inner contour) with or without specular light reflection, and the second devoted to detect the iris circle detection (outer contour) with or without specular light reflection.



Fig. 1. Circular form of the Iris and pupil

\subsection{Training Process}

The iris training set consisted of 100 labeled pupil and 100 labeled iris images taken from the UBIRIS and CASIA databases. The region training set was manually prepared by means of the selection of the rectangular regions, and then these samples were saved and rescaled all to a size of 24 x 24 pixels. (Fig. 2). In the training phase, the classifier was exhaustively trained using these sets of regions by the cascade detectors in a wide variety of training images. 


\subsection{Description of the Automatic Detection Process}

The process begins with the pupil border detection by means of the cascade detector specialized in the pupil detection with specular light reflection or without it (1). As a result, the coordinates of the tangential square that contains the pupil are obtained. A second cascade detector is triggered to look for the iris circle, as in the previous step we obtain the coordinates of the tangential square that contains the iris (2).



Fig. 2. Region selection for iris training samples in the UBIRIS database

\section{Approximation of Pupil and Iris Contour to a Circle Inscribed in a Square}

The iris inner contour coincides with the external pupil boundary. Since it is assumed that the pupil and iris possess circular form, the parameters that should be obtained are, the pupil centre coordinates and its radio. To solve this task the algorithm of tangential square circle was designed.

The general idea is very simple, beginning with an automatic localized tangential square; we will calculate the radius of the circumference and the centre of the square that is the centre of the pupil or iris. With the centre and the radius we draw a bounded circumference to the square. The calculated circumference is the pupil or iris contour.

The steps for the proposed approximation are as follows:

Step1: Find the radius of the circumferences

The radius of the circumferences is calculated by expressions:

$$
r_{p, i}=\frac{m x_{p, i}-n x_{p, i}}{2}=\frac{m y_{p, i}-n y_{p, i}}{2}
$$

Where $n x_{p, i}$ and $m x_{p, i}$ are the extreme coordinates of the horizontal or vertical side of the square for the $\operatorname{pupil}(p)$ and $\operatorname{iris}(i)$. (Fig. 3)

Step 2: Find the centres of the tangential squares.

The centres of the squares $\mathrm{C}_{\mathrm{p}}\left(\mathrm{x}_{\mathrm{p}}, \mathrm{y}_{\mathrm{p}}\right)$ and $\mathrm{C}_{\mathrm{i}}\left(\mathrm{x}_{\mathrm{i}}, \mathrm{y}_{\mathrm{i}}\right)$ are calculated by expressions :

$$
\begin{aligned}
& x_{p, i}=n x_{p, i}+r_{p, i} \\
& y_{p, i}=n y_{p, i}+r_{p, i}
\end{aligned}
$$


Where $x_{p, i}$ and $y_{p, i}$ are the horizontal and vertical coordinates of the square centres of the pupil $(p)$ and iris $(i)$ respectively (Fig. 4). The mean centre $\left[C_{m}\left(x_{m}, y_{m}\right)\right]$ of the eye is calculated by the expression:

$$
x_{m}=\frac{x_{p}+x_{i}}{2} \quad y_{m}=\frac{y_{p}+y_{i}}{2}
$$

Step 3: With the circumference parameters we automatically draw the bounded circumference to the square that is the pupil $(p)$ or iris $(i)$ contour by expression.

$$
x_{p, i}=r_{p, i} \cdot \cos \theta, y_{p, i}=r_{p, i} \cdot \operatorname{sen} \theta
$$

Where $x_{p, i}$ and $y_{p, i}$ are the coordinates of the circumferential sector inner and outer borders of the texture codification area.
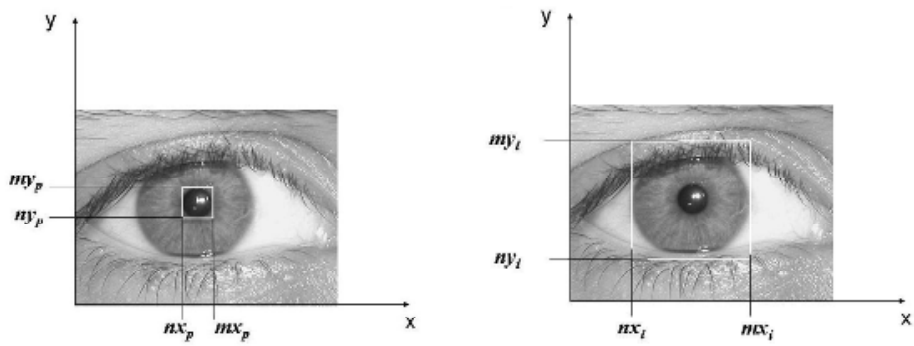

Fig. 3. Coordinates of the squares and centres of the circles

\subsection{Lookup Table}

To increase the speed of the proposed algorithm we put all the possible values of the circumference coordinates in a lookup table. A lookup table also contents the all possible values of the points $\mathrm{x}_{\mathrm{im}}, \mathrm{y}_{\mathrm{im}}$ and $\mathrm{x}_{\mathrm{pm}}, \mathrm{y}_{\mathrm{pm}}$ that correspond to all possible values of $r_{i m}$ and $r_{p m}$ respectively and for all possible values of the $\theta$ angles in the intervals $\left[315^{\circ}-45^{\circ}\right]$ and $\left[135^{\circ}-225^{\circ}\right]$.

\subsection{The Pupil Pixel Value Change}

In a previous work [7] we presented an algorithm that we called "Three points algorithm". The proposed algorithm takes advantage of the pupil circular form using elements of analytic geometry, in particular of the determination of the bounded circumference to a triangle. To obtain the parameters that define the external contour the Daugman algorithm [1,2] was used. This second algorithm receives the output from the first one and after that, searches the abrupt gradient changes of a contour integral to find the iris border. The problem of this proposal was the fact that its effectiveness depends of the quantitative texture feature, as the standard deviation to detect the frontier between the pupil and the iris. When we work on a database with well controlled conditions like the CASIA database [3] composed by images taken with near infrared illumination, without the presence of specular light the 
performance of the combination of these two algorithms is very high, but when the images are affected by specular light it fails due to the high variability detected in the pupil. To avoid this problem and improve the performance of this combination we implemented an optional step in our new proposal

Step 4: Substitution of the pixel values inside the pupil circumference by zero values, this guarantees that the performance of Daugman algorithm will be effective because the difference between the standard deviation within the pupil and in the iris area increase abruptly.


Fig. 4. Determination of the circular sector of the texture codification area for feature extraction

\section{Experiments}

For a complete evaluation of the performance of our proposal we divided the experimental process in two parts, the first one oriented to the measurement of the effectiveness of the automatic detection of the tangential square enclosing the iris inner and outer contours, and the second one oriented to measure the accuracy of determination of the texture codification area for feature extraction and identification.

\subsection{Measurement of the Effectiveness in the Automatic Tangential Square Detection}

We defined a set of measures necessary to know accurately the effectiveness behaviour of the detector according to the final mission of the process. They are:

ESD (External Square Difference): The difference in area between optimal square containing the real contour and the one automatically detected if the area of the square detected is bigger (Fig 5, left), it is given in percentage and calculated by the expression: 


$$
E S D=\frac{\sum(D A S-T A S)}{\sum D A S .100}
$$

where $D A S$ and $T A S$ are the areas of the tangential square automatically detected and the area of the actual tangential square respectively.

ISD (Internal Square Difference): The difference in area between optimal square containing the actual contour and the one automatically detected if the area of the detected square is smaller (Fig 5, right), it is given in percentages and calculated by the expression:

$$
I S D=\frac{\sum(T A S-D A S)}{\sum T A S .100}
$$

GFT (General effectiveness of automatic detection) is the general percentages of accuracy taking in to account the ISD and ESD, and expresses the precision with which the detector is able to enclose the actual contour. It is calculated by the expression.

$$
G F T=100-\left[\frac{1}{2}(E S D+I S D)\right]
$$

We also measured the effectiveness of the automatic detection process by computing the correctly detected regions, missed regions and falsely detected regions.

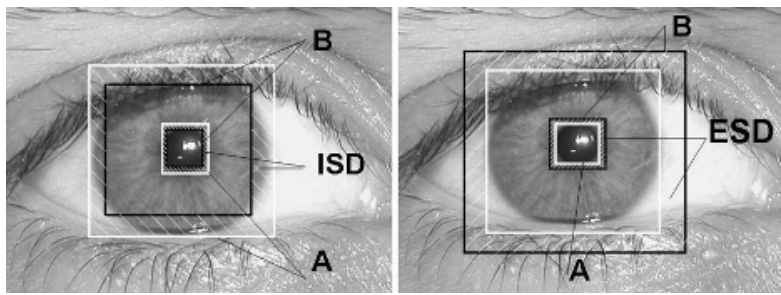

Fig. 5. Measurement of the effectiveness of the automatic iris inner and outer contour detection. A: Optimal square containing the actual contour, B: Automatically detected square.

\subsection{The UBIRIS Database}

We did the experiments in the UBIRIS database[11]. UBIRIS database is composed of 1877 images collected from 241 persons during September, 2004 in two distinct sessions. Its main characteristic results from the fact that, in opposition to the existing public and free databases (CASIA and UPOL), it incorporates images with several noise factors, thus permitting the evaluation of robustness iris recognition methodologies. For our experiment we took 2 images per person (482 images of 241 persons) and divided the database in two sections, training set, 100 persons (200 images) and test set, 141 persons (282 images), see Fig.7. 


\subsection{Measurement of the Accuracy in Determination of the Texture Codification Area for Feature Extraction and Identification}

The idea was to measure the similarity between images that contain the texture codification area for feature extraction and identification, comparing images generated from the detected automatically contours and the ones detected by Daugman algorithm(Fig 6). As a similarity measure we used normalized correlation. Normalized correlation has been chosen as it has proved to be a successful similarity measure in computer vision. For identical images it takes the maximum value equal to unity, it is defined as:

$$
N c=\frac{\sum_{i} \sum_{j}\left(w(i, j) w_{1}(i, j)\right)}{\sqrt{\sum_{i} \sum_{j} w(i, j)^{2} \sum_{i} \sum_{j} w_{1}(i, j)^{2}}}
$$

Where the first image is $w(i, j)$, the second image is $w_{1}(i, j)$.

For this experiment we used images from CASIA database. We compared 108 images generated from our approach against 108 images of the same individuals generated from Daugman algorithm.

The same procedure we used to compare images from UBIRIS database. For this experiment we compared the $N c$ with the radial difference in pixels between outer contours generated by our approach and the ones generated by Daugman algorithm with previous inner contour detection and normalization of pupil pixel values using our proposed approach (3.2).


Extracted texture codification areas

Fig. 6. Extraction of the texture codification area for feature extraction and identification

\subsection{The Time Consuming Evaluation}

For the evaluation of time consuming in the detection process we compared the time taken for the automatic contour detection using our approach and the time taken using the proposed by Daugman detection algorithm with the same quantity of images and similar hardware configuration. We expressed these measures in milliseconds related to the time consumed for contour detection by Daugman algorithm in 100 images from CASIA database. 


\subsection{The CASIA Database}

The CASIA Iris Image Database [3] includes 108 classes and each class has 7 iris images captured in two sessions with a time interval about a month. So there are totally 756 iris images with a resolution of 320x280 pixels. In our experiment we used 1 image per class (108 images), see Fig 8.

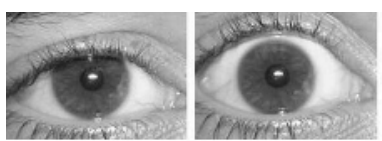

UBIRIS Database

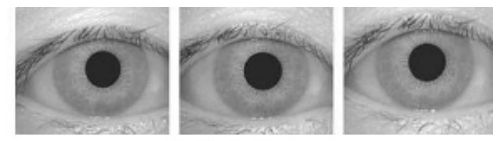

CASIA Database

Fig. 7. Samples of the CASIA database used in the experiment

\section{Experimental Results}

In Table 1 and 2 we present the results obtained by experimental procedure described in 4.1 in the UBIRIS database. We can see that in both cases, ERD and IRD are less than $2.0 \%$ and the GFT is bigger than $98 \%$. The detector only fails in 3 samples detecting the inner contour $(0.62 \%)$ but in 20 samples detecting the outer contour $(4 \%)$.

Table 1. Values of the ERD and IRD in the UBIRIS database

\begin{tabular}{|c|c|c|c|}
\hline \multicolumn{2}{|c|}{ Inner Iris contour } & \multicolumn{2}{c|}{ Outer Iris contour } \\
\hline IRD (\%) & ERD (\%) & IRD (\%) & ERD (\%) \\
\hline 0.64 & 0.33 & 1.65 & 0.98 \\
\hline \multicolumn{2}{|c|}{ GFT (\%) } & \multicolumn{2}{c|}{ GFT (\%) } \\
\hline \multicolumn{2}{|c|}{99.51} & \multicolumn{2}{c|}{98} \\
\hline
\end{tabular}

Table 2. Performance of the Detector

\begin{tabular}{|c|c|c|}
\hline Correctly Detected (\%) & Missed (\%) & Falsely Detected(\%) \\
\hline \multicolumn{3}{|c|}{ Pupil Contour (Inner) } \\
\hline 99.3 & 0.7 & 0.05 \\
\hline \multicolumn{3}{|c|}{ Iris-Contour (Outer) } \\
\hline 95.9 & 4.1 & 0 \\
\hline
\end{tabular}

In the Fig. 8 we present the results obtained by experimental procedure described in 4.3. The normalized correlation coefficients $(\mathrm{Nc})$ are in all the cases of the CASIA database (Fig.8A) bigger than 0.9. This result shows that the texture codification areas extracted from images in which the iris contour was automatically detected by our own approach are very similar to the ones obtained from those in which the iris contour was detected by Daugman algorithm. They can be used for the feature extraction and identification. The results in the UBIRIS database (Fig.8B) show that the under not controlled illumination conditions the $N c$ values are in most of cases bigger than 0.7 . 
From Fig.9, we observe that when our approach is used, the time of processing decreases in approximately $45 \%$ of the total of the time used by Daugman algorithm to process the same quantity of images with similar hardware configuration.
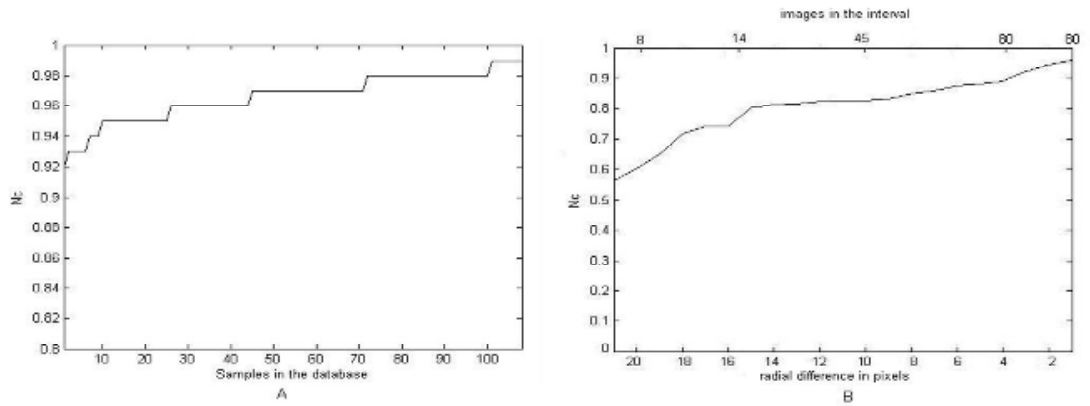

Fig. 8. Comparison of the normalized correlations: A: in the CASIA database, B: in the UBIRIS database

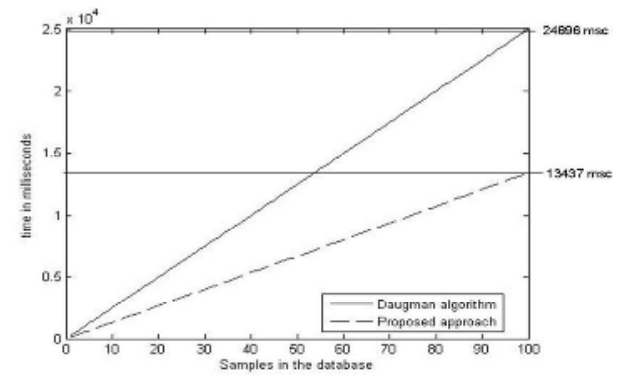

Fig. 9. Comparison of the time consuming (in milliseconds) using Daugman algorithm against proposed approach in the CASIA database

\section{Conclusions}

In this paper we proposed an approach based on the Adaboosting technique for fast detection of the iris and pupil contour in the images of human eyes. The proposed approach overcomes the problem of the specular light reflected in the pupil or in the iris and allows the detection and localization of the circular sector of the texture codification area for feature extraction. To increase the speed of the proposed algorithm we used the Lookup table containing all the possible values of the circumferential sectors describing the iris contours. The experimental results show that our proposal is equivalent in accuracy in comparison with the well validated Daugman algorithm together with a relevant improvement of the computational time.

\section{Acknowledgements}

We want to thank the National Laboratory of Pattern Recognition from Beijing, China and the "Departamento de Informática da Universidade da Beira Interior, Covilhã, 
Protugal", for its generosity in allowing us the use of the CASIA and UBIRIS Iris Images Data Bases in our experiments.

\section{References}

1. Daugman, J.: High Confidence Visual Recognition of Persons by a Test of Statistical Independence. IEEE Transactions on Pattern Analysis and Machine Intelligence. Vol. 15. No 11. 1148-1160 pp., (1993).

2. Daugman, J.: Demodulation by Complex-Valued Wavelets for Stochastic Pattern Recognition. International Journal of Wavelets, Multiresolution and Information Processing. Vol. 1. No. 1. 1-17 pp., (2003).

3. Center for Biometrics and Security Research (CBSR). CASIA Iris Image Database, http://www.sinobiometrics.com.

4. Cui, J., Y., Wang, T. Tan, L. Ma, Z. Sun: A Fast and Robust Iris Localization Method Based on Texture Segmentation, Center for Biometric Authentication and Testing, National $\backslash$ Laboratory of Pattern Recognition. Disponible en internet. (2005)

5. Wildes, R.: Iris Recognition: An Emerging Biometric Technology, Proceedings of the IEEE, Vol. 85, pp.1348-1363, (1997).

6. Camus, T. A., R. Wildes: Reliable and Fast Eye Finding in Close-up Images, Proceedings of the IEEE International Conference on Pattern Recognition, (2002).

7. Gil J L , and Díaz Y. A New Method for Iris Pupil Contour Delimitation and its Application in Iris Texture Parameter Estimation,: CIARP 2005, LNCS 3773, pp. 631 641, 2005. Springer-Verlag Berlin Heidelberg 2005

8. Center for Biometrics and Security Research (CBSR). CASIA Iris Image Database, http://www.sinobiometrics.com.

9. Viola P. and Jones M. Rapid Object Detection Using a Boosted Cascade of Simple Features. Mitsubishi Electric Research Laboratories, Inc., 2004201 Broadway, Cambridge, Massachusetts 02139.

10. Lienhart Rainer. Haarcascade_frontalface_default.xml. Intel License Agreement For Open Source Computer Vision Library. 2000.

11. UBIRIS Database. Departamento de Informática da Universidade da Beira Interior, URL: http://iris.di.ubi.pt/. 